\title{
Turbulent compressible convection with rotation
}

\author{
Nicholas H. Brummell ${ }^{1}$ \\ ${ }^{1}$ JILA/Department of Astrophysical and Planetary Sciences, University of Colorado, Boulder, \\ CO 80309-0440, USA \\ email: brummell@solarz.colorado.edu
}

\begin{abstract}
We examine the effect of rotation on turbulent compressible penetrating convection, motivated by the dynamics of the solar interior. We find that rotation can influence large-scale coherent structures in the turbulent flow whilst leaving the small-scales isotropic. The dynamics in this case are very different from more laminar situations where all scales are rotationallyinfluenced. This may hint as to why full models of the global solar differential rotation are difficult.
\end{abstract}

Keywords. Convection, Sun: rotation

\section{Introduction}

The outer $30 \%$ of the Sun transports heat by convection. This outer convection zone overlies a radiative interior that is stable to convection. This solar interior is not in rigid body rotation but rather rotates differentially, as revealed by helioseismology (see e.g. Thompson, Christensen-Dalsgaard, Miesch \& Toomre (2003)). The convection zone appears to rotate constantly on almost radial cones, whereas the inner radiative zone rotates rigidly. The two regions are joined by a narrow interface of strong radial shear, known as the tachocline. A major quest in solar physics has been to understand how this rotation profile is generated. Motions on many scales appear in the convection, from small (diameter $\approx 1000 \mathrm{~km}$ ) granules, through large supergranules $(\approx 30,000 \mathrm{~km})$ to giant cells and other structures on the order of the largest scales $(\approx 200 \mathrm{Mm})$. The timescales are such that rotation influences motions at least down to the scale of supergranules. So an interesting question for the solar context is, what is the influence of rotation on turbulent convection?

The solar interior is compressible. Furthermore, motions generated in the convection zone are not absolutely confined there, and may continue somewhat into the stable region. Thus the convection overshoots or penetrates, terms often used interchangeably, but technically differing depending on how effective the thermodynamic mixing is in the initially stable region. We therefore study the effect of rotation on turbulent, compressible, penetrative convection. Obviously, the Sun operates in a spherical geometry, and the dynamics in this natural geometry are indeed studied (e.g. Miesch, Brun \& Toomre (2006)). However, in pursuing this global geometry, the finite computing resources are utilised for the large scales, since the largest scale must necessarily be included. This presents a problem since there are not enough resources to cover the full spectrum of turbulent scales down to the realistic diffusion scale. The best spherical shell simulations currently simulate the global scale down to about the supergranular scales. An issue then arises. By the construction of the model, diffusion must act at the supergranular scales, which is still a rotationally-constrained scale. What happens when the diffusive scales 


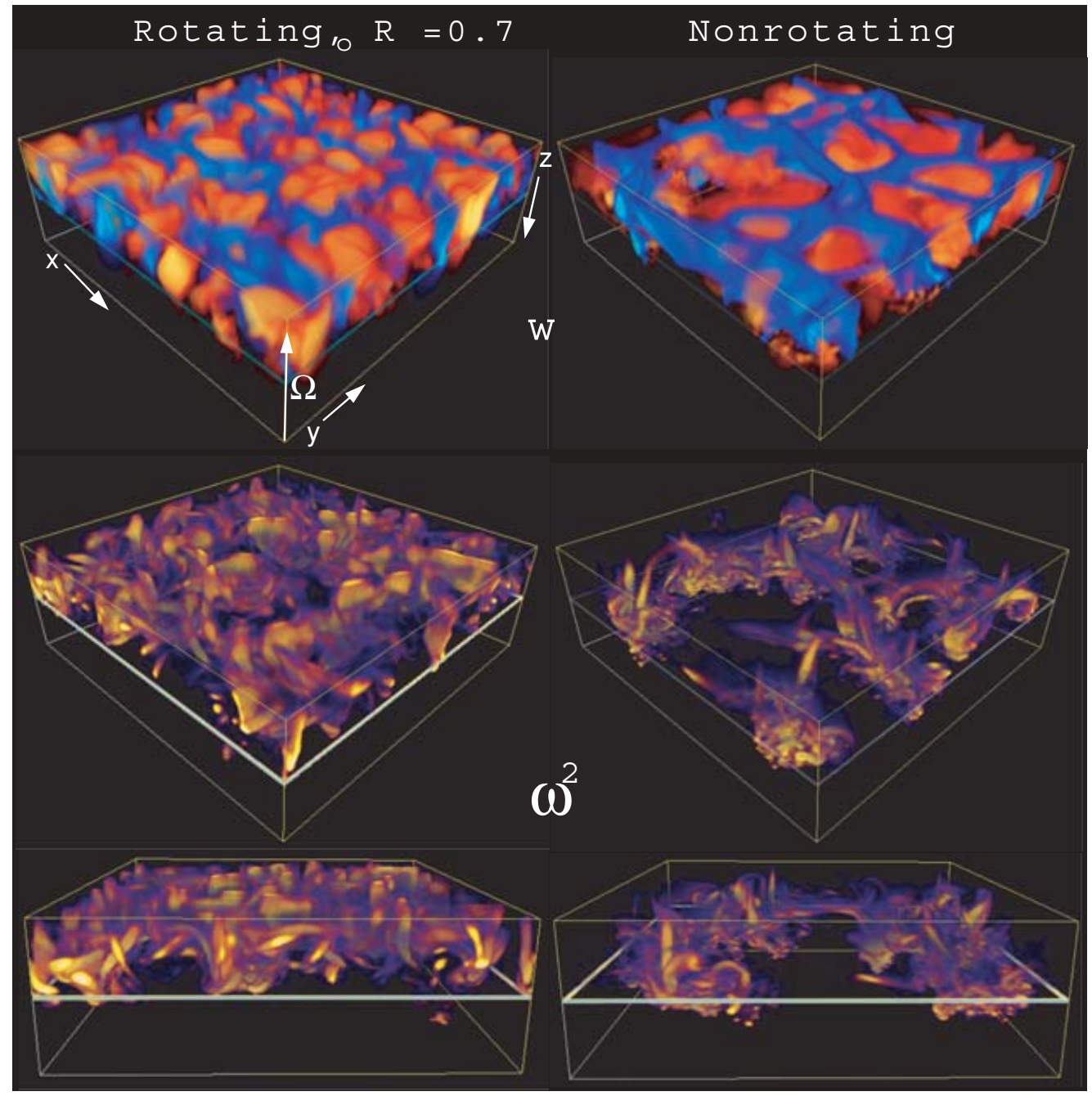

Figure 1. A comparison of rotating and non-rotating penetrative convection. Volume renderings of the vertical velocity (top panels) and the enstrophy density (other panels). Upwards velocities are red and yellow hues, whereas downwards velocities are blues. Strong vorticity is yellow. Weak values of any field are more translucent. [From Brummell, Clune \& Toomre (2002)]

are separated from the rotationally-constrained scales? Is this a dynamically different situation?

In an attempt to address this issue, we study the influence of rotation on turbulent compressible penetrative convection in a Cartesian domain. This local approach allows the maximum resolved scale (the domain size) to be a chosen parameter, and so our finite numerical degrees of freedom can be applied to the smallest scales, from the diffusive scale to the local domain scale. We then choose the rotation rate so that the larger of these scales are rotationally-influenced, but the smaller are not (measured by the Rossby number Ro calculated for the largest scale). We proceed in this manner, allowing further the rotation vector to be inclined to the vertical to simulate the placement of the local domain at various latitudes. 


\section{The influence of rotation on penetrative convection}

The typical forms of non-rotating and rotating penetrative compressible convection are shown in the snapshots of Figure 1. This figure shows volume renderings of the vertical velocity, $w$ (uppermost panels) and the enstrophy density (vorticity squared, $\omega^{2}$; other panels), at a single time for two typical cases with and without rotation. The Rayleigh number has been carefully chosen so that the degree of supercriticality is similar in both cases (for details see Brummell, Clune \& Toomre (2002)). The motions in the upper convective half of the domain have the general form of asymmetric convection, with the asymmetry arising from the background density stratification (which varies by a factor of 11 over the convection zone, and a factor of 45 over the whole layer). Upward motions are slow and broad in scale, whereas downward motions are concentrated into a cellular network at the top, and with depth are dominated by downflowing plumes emanating from the interstices of that upper network. These plumes are coherent structures amongst the turbulence, since they remain roughly in place for a few integral turnover times. They play a dominant role in the transport dynamics (see e.g. Cattaneo, Brummell, Toomre, Malagoli \& Hurlburt (1991)). The convectively-driven plumes are not confined to the upper convective layer, but may continue their downward progress beyond the unstablestable interface (at the half-depth point in this domain) for some distance. In these cases, although the Peclet number is large, the highly intermittent nature of the plumes in space and time means that the thermodynamic mixing is not strong enough to extend the adiabatic convective interior below the interface. Thus, the motions are technically "overshooting" as opposed to "penetration" (Zahn (1991)). The depth of the overshooting is dependent on many parameters (for a full discussion, see Brummell, Clune \& Toomre (2002)).

The left column of Figure 1 shows a case with significant rotational influence, where in this case the rotation is about a vertical axis (as if the domain were positioned at the north pole), with a convective Rossby number, $R o=\sqrt{R a / \sigma T a}=0.7$ (where $\sigma$ is the Prandtl number and $T a$ is the Taylor number; $R o$ represents the ratio of the rotation time to a convective turnover time). A number of effects are immediately apparent. Firstly, the overall scale of the convection is reduced. The cells of the network near the top, seen most clearly in the velocity, are obviously smaller. This is a well-known effect due to the deflecting action of the Coriolis force. Secondly, most apparent in the enstrophy renderings, the rotating case appears much "busier", i.e. the amount of smallscale, strong, turbulent vorticity appears to be greater and seems to fill more volume of the domain. The inclusion of rotation also tends to make the flow more isotropic at the smaller scales. These are results due to the enhanced horizontal mixing engendered by the rotation and the availability of the pool of background rotational vorticity from which to draw. Furthermore, with rotation, the downflows must all have the same sense of vertical vorticity (since they are "spun up" in the convergence that creates them) and so vortex-vortex interactions between the plumes occur, enhancing horizontal mixing and isotropy.

\section{The effects of rotation and latitude: turbulent alignment of coherent structures amongst the turbulence}

We can effectively vary the position of the Cartesian domain through different latitudes by tilting the rotation vector away from the vertical (aligned with gravity) in the $y-z$ (north-down) plane. Figure 2 shows three cases at the same parameters but at different latitudes $\phi=45^{\circ}, 15^{\circ}$ and $0^{\circ}$ (the equator). The main thing to notice from 


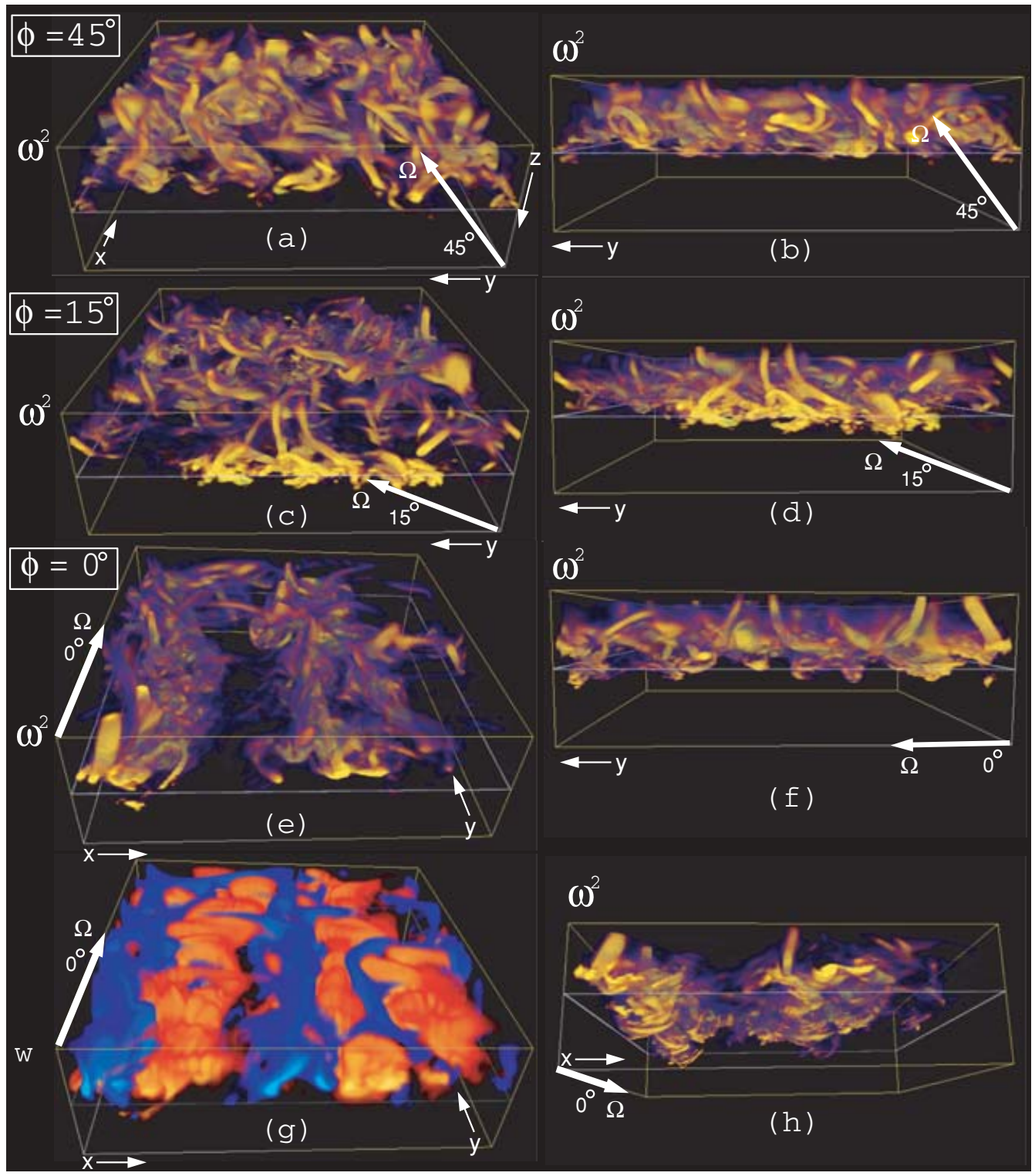

Figure 2. Snapshots of rotating penetrative compressible convection at different latitudes. Shown are volume renderings (as in Fig. 1) of the enstrophy density $\omega^{2}$ from two different angles for three latitudes, $\phi=45^{\circ}, 15^{\circ}, 0^{\circ}$ (degrees north). Panel $(g)$ shows the vertical velocity of the solution at the equator, $\phi=0^{\circ}$. [From Brummell, Clune \& Toomre (2002)]

these renderings is that the large-scale features - the coherent structures of the turbulent flow - appear to align themselves with the rotation vector in all but the lowest latitude case. Obviously the alignment is not perfect, since buoyancy will try and counteract this behaviour and make the flows move vertically, and also the boundary conditions are such that the vorticity must be purely vertical at the boundaries. However, the effect is clearly apparent: alignment is seen over the length of the coherent structure including its transit across the interface and continuance into the stable layer. The small-scale turbulent 

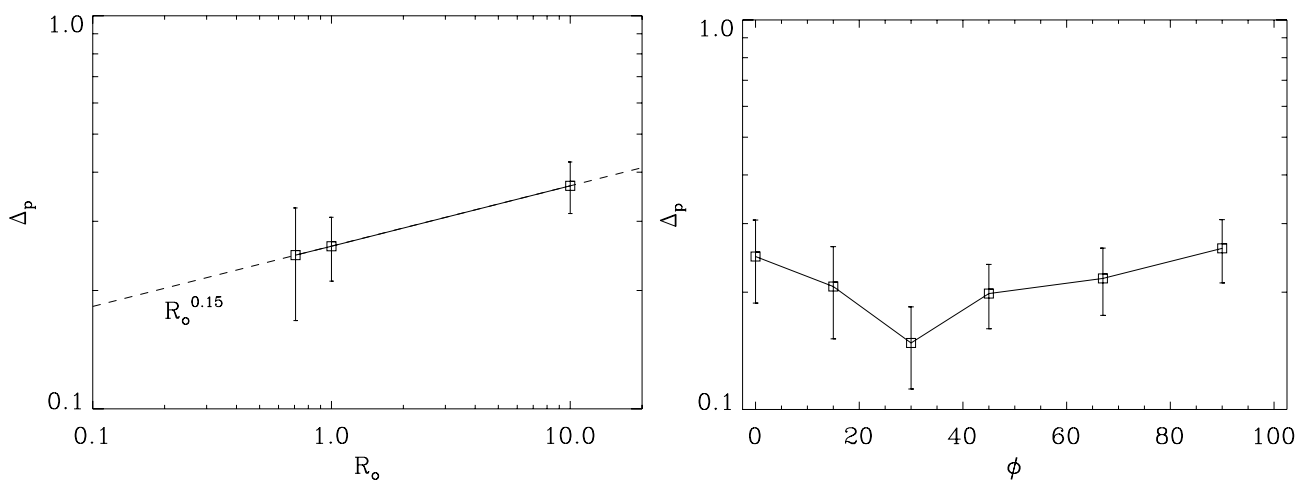

Figure 3. (a) A plot of the average penetration depth $\Delta_{p}$ against rotational influence measured by the Rossby number Ro. (b) The variation of $\Delta_{p}$ with latitude $\phi$. [From Brummell, Clune \& Toomre (2002)]

motions are too quick to sense the rotation and decouple, but the larger-scale temporally and spatially coherent structures are affected.

At the lowest latitude, $\phi=0^{\circ}$, when the domain is positioned at the equator, the effects of buoyancy and the turbulent alignment of the coherent structures act perpendicular to each other. A slightly altered geometry of the convection results. Convective roll-like behaviour is set up, with the axes of the pseudo-rolls aligned with the rotation (the $y$-direction, north). Turbulent vorticity is still present but confined to bands of upflow and downflow along the north-south edges of the pseudo-rolls. The turbulent alignement mechanism is likely replaced by this mode since it is more efficient than trying to force the downflows to be nearly horizontal.

\section{The effects of rotation and latitude: variation of the penetration depth}

Figures $3 \mathrm{a}$ and $3 \mathrm{~b}$ show the effect of the variation of rotational influence at a fixed latitude, and the effect of the variation of latitude at a fixed rotational influence, respectively. Figure 3a shows the average penetration depth $\Delta_{p}$ of the overshooting motions measured for three simulations that are identical except for the rotational influence, which varies through $R o=10,1,0.7$. Clearly the depth of penetration of the convective motions decreases with increasing rotational influence (decreasing $R o$ ). This can be attributed to an increased degree of horizontal mixing for higher rotational influence, as mentioned above, reducing the vertical velocities in the downflows and therefore the overshooting. For example, for $R o=0.7$, the rms velocities in the downflows are $60 \%$ of those in the non-rotating case.

Figure $3 \mathrm{~b}$ shows simulations that are identical with $R o=1.0$ but for latitudes varying through $\phi=0^{\circ}, 15^{\circ}, 30^{\circ}, 45^{\circ}, 67^{\circ}, 90^{\circ}$. The dependence of the measured average penetration depth $\Delta_{p}$ is not monotonic, decreasing from a maximum at $\phi=90^{\circ}$ to a minimum at $\phi \approx 30^{\circ}$ then increasing somewhat at the lowest latitudes $\left(\phi=0^{\circ}, 15^{\circ}\right)$. The initial decrease in the penetration depth as latitude decreases away from the north pole can be explained by the turbulent alignment of the coherent structures, since these are the plumes that do the overshooting. At latitudes away from purely north $\left(\phi<90^{\circ}\right)$, the penetration of these structures is angled to align with the rotation vector and therefore does not penetrate so far. This effect is interrupted at low latitudes by the change in the geometry of the convection at low latitudes mentioned above. At the lower latitudes, 


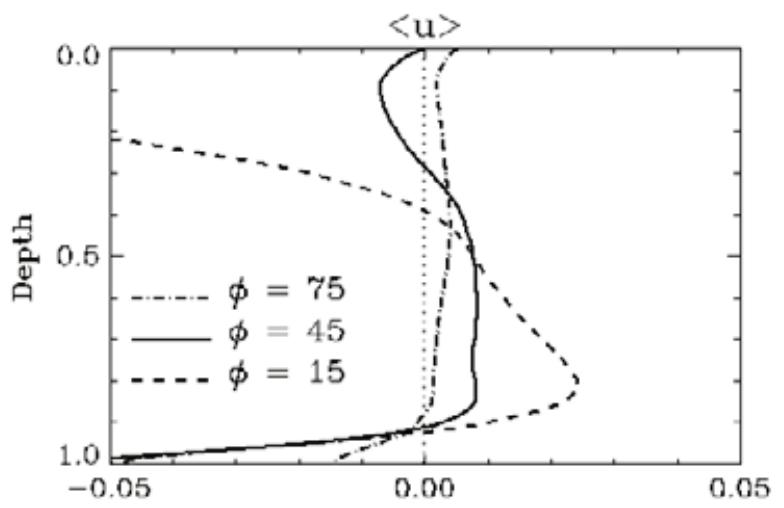

Figure 4. A plot of the mean zonal velocity $\langle u(z)\rangle$ against depth $z$ for three different latitudes, $\phi=15^{\circ}, 45^{\circ}, 75^{\circ}$. [From Brummell, Hurlburt \& Toomre (1998)]

the competition of the rotational alignment against buoyancy leads to pseudo roll-like convective cells rather than turbulence punctuated by coherent structures. This new geometry penetrates a little further due to the large filling factor of the downward motions and the "flywheeling" effect of the more laminar overturning roll cells.

\section{The effects of rotation and latitude: differential rotation}

The equivalent of the differential rotation at a given latitude in the local Cartesian model is the horizontally-averaged and time-averaged ("mean") zonal (east-west) velocity as a function of depth, $\langle u(z)\rangle$. Figure 5 shows this quantity for three non-penetrating simulations with similar parameters except at varying latitudes $\phi=15^{\circ}, 45^{\circ}, 75^{\circ}$. The general form of the solutions is that the zonal flow has strong variations near the boundaries, but is roughly constant in the interior. This is reminiscent of the "nearly constant on radii" helioseismic inferences. The interior strength of the flows increases with decreasing latitude. These effects can be understood in terms of the Reynolds stresses that the turbulent flows induce. Reynolds stresses are source terms in the equations for the generation of the mean flows resulting from correlations between the vertical velocity and the horizontal velocity components. A steady zonal flow $\langle u(z)\rangle$ is generated by correlations between the vertical velocity $w$ and the meridional flow $v$. In the topology of the turbulent compressible convective flows described above, the small-scale motions are generally isotropic and de-correlated. However, motions down the coherent downflowing structures can provide significant correlation between $w$ and $v$ when those structures are tilted in the $y-z$ plane to align with the rotation. At lower latitudes, where the tilt is more pronounced, the correlation is stronger, and so the mean flows are enhanced. Near the north pole $\phi=90^{\circ}$ the correlation is lost and the mean flows are weak. The results for turbulent flows are very different from laminar flows. Laminar flows by definition are smooth on scales that are all influenced by the rotation (or all not influenced, depending on their turnover time compared to the rotation time). For turbulent flows, many of the scales present may not feel the rotation, since they turnover too quickly, whereas larger-scale coherent components of the flow may be affected.

\section{Conclusions}

We have examined the effect of rotation on penetrative (or, more technically correct, "overshooting") compressible turbulent convection. Rotation influences the nature of the 
convection: the overall scales are reduced, the small-scales are made more isotropic whilst the larger scale coherent structures are aligned with the rotation vector, and horizontal mixing is enhanced at the expense of vertical mixing. The degree of overshooting is affected by the rotational transfer of vertical motions into horizontal flows, and by the latitudinal positioning of the model due to the tilting of the coherent downflows.

We see that the rotational influence on a turbulent flow depends on the range of scales present. If the range is sufficiently large, then there are scales at the top end that are significantly affected by the rotation, but there are also small scales at the bottom end of the spectrum that are isotropic and unaffected by Coriolis forces. We expect diffusion to act at the smallest scales where things are isotropic. Current global models of shell convection that are attempting to reproduce and interpret the cause of the solar differential rotation have insufficient resolution to be able to span the full range of scales so that the three scale regimes ( 1 - rotationally-influenced; 2 - isotropic rotationally-notinfluenced; 3 - diffusive) are separated. When the diffusive regime and the rotationallyinfluenced regime overlap and there is no isotropic regime, the dynamics of the flows and in particular Reynolds stresses may be different. This may account for why it has been so hard to reproduce the solar differential rotation in any robust parameter regime of current spherical shell models (Miesch, Brun \& Toomre (2006)).

\section{Acknowledgements}

The author would like to thank his co-authors involved in this work: Tom Clune, Neal Hurlburt and Juri Toomre. The author would also like to thank the organisers of the session for the invitation.

\section{References}

Basu, S., Anita, H.M. \& Narasimha, D. 1994, MNRAS 267, 209

Brummell, N.H., Clune, T.L. \& Toomre, J. 2002, ApJ 570, 825

Brummell, N.H., Hurlburt, N.E. \& Toomre, J. 1998, ApJ 492, 955

Cattaneo, F., Brummell, N.H., Toomre, J., Malagoli, A. \& Hurlburt, N.E. 1991, ApJ 370, 282

Miesch, M.S., Brun, A.S. \& Toomre, J. 2006, ApJ 641, 618

Monteiro, M.J.P.F.G., Christensen-Dalsgaard, J. \& Thompson, M. 1994, A\&SA 283, 247

Roxburgh, I.W. \& Vorontsov, S.V. 1994, MNRAS 268, 880

Thompson, M., Christensen-Dalsgaard, J., Miesch, M.S. \& Toomre, J. 2003, ARAA 41, 599

Tobias, S.M., Brummell, N.H., Clune, T.L. \& Toomre, J. 2001, ApJ 549, 1183

Zahn, J.-P. 1991, A\&SA 252, 179

\section{Discussion}

R.F. STEIn: Did you consider using a Kramers type thermal conductivity instead of a function of depth?

N.H. BRUMmell: Yes, we have considered that. Using a fully three-dimensional thermal conductivity makes the thermal diffusion term numerically nonlinear, which is a pain for pseudo-spectral codes like ours. This is not a problem for finite-difference codes of course. David Porter and Paul Woodward did some comparison runs to ours on the same problem using a PPM finite-difference method and a Kramers Law thermal conductivity. They saw very similar results. At some stage, I will implement a thermal conductivity as a function of the mean thermodynamic variables rather than just depth to see if this makes any difference, but I expect the results to be qualitatively the same. 
R.F. Stein: Since you take the thermal conductivity a function of depth rather than temperature and density I would not expect to see any change in the depth of the adiabatic layer.

N.H. BRummell: This is a common misconception. The adiabatic extension of the convective region is not precluded just because the thermal conductivity changes nature at a particular depth. Two-dimensional simulations show very clearly an extended adiabatic region due to true penetration when $\kappa=\kappa(z)$ only. The lack of adiabatic extension in the 3-D case is due to physical dynamic processes, most likely the low filling fact of downflowing plumes. You even see this lack of extension in your simulations, Bob!

R.F. Stein: You have these vorticity funnels in the upper part of the domain. These don't look twisted up and turbulent. In our simulation we see these very twisted up in the convective region and only funnel like in the photosphere.

N.H. BRUMMELL: I think our simulations are at a degree of turbulence where the interior is small-scale turbulence but the large scale downflowing structures are not ripped apart greatly by shear instabilities. At higher Reynolds numbers, I would expect the downflows to become more turbulent too. The simulations that I mentioned of Porter and Woodward were at much higher effective Reynolds number, since PPM is formerly an inviscid scheme and the viscosity is all numerical. These showed incredibly complex downflowing structures, full of small-scale vorticity. However, the behaviour was very similar to our simulations in terms of the average fluxes, despite the great complexity.

DAVID ARnetT: Can we quantify the disagreement with helioseismology regarding a sharp change (or discontinuity) at the base of the convection zone?

N.H. Brummell: This question should be addressed by the helioseismologists, but is my understanding from the literature that there is no real observational evidence for a sharp transition at the base of the convection zone (Basu, Anita \& Narasimha (1994); Monteiro, Christensen-Dalsgaard \& Thompson (1994); Roxburgh \& Vorontsov (1994)). Furthermore, the helioseismic inversion kernals are insufficient to resolve a sharp transition at that depth. Our simulations are actually consistent at least with all these conclusions!

F. Busse: How many scale heights were used in the simulations? Shouldn't one see also effects of magnetic buoyancy?

N.H. BRummelL: [This question is in relation to magnetic pumping which I discussed in the talk but not in this paper. Magnetic pumping is the term that describes the fact that compressible penetrative convection redistributes a mean magnetic field so that the majority of it resides in the overshoot zone. See Tobias, Brummell, Clune \& Toomre (2001) if interested.] The simulation domain encompasses about 10 pressure scale heights, or a density contrast of 20 or so across the convective layer in the relaxed state. Yes, this is considerable and therefore when the mean magnetic field is released it does rise due to magnetic buoyancy. However, the pumping effect is significant enough to overcome this. The downflows are strong and their advective effect carries field to the overshoot zone against their buoyancy. In the redistributed statistically-steady state of the mean magnetic field, field elements are constantly rising due to magnetic buoyancy and descending due to advection by the downflows to maintain the state. 